\title{
NUEVA MUJER Y NUEVAS ELECTRAS: APROXIMACIONES A LA MITOLOGÍA CLÁSICA EN EL TEATRO BURLESCO VICTORIANO ${ }^{1}$
}

\author{
Laura Monrós Gaspar, Universidad de Valencia ${ }^{2}$ \\ Email: laura.monros@uv.es
}

\begin{abstract}
This paper seeks to analyze Francis Talfourd's Electra in a New Electric Light (1859) as related to the Victorian stereotype of the strong-minded woman. After a brief introduction on the links between nineteenth-century burlesque and the social history of women in Victorian times, I shall focus on the figure of Electra as epitome of late nineteenth-century representations of New Women.

Keywords: Burlesque, Electra, strong-minded woman, Victorian.

Title in English: New Woman and New Electras: Approaches to Classical Mythology in Victorian Burlesque Theatre.

Resumen: Resumen: El objetivo de este trabajo es el estudio de Electra in a New Electric Light (1859) de Francis Talfourd a partir del estereotipo victoriano de la strong-minded woman. Para ello, tras comentar la relación existente entre el teatro burlesco decimonónico de tema clásico y la historia social de la mujer a lo largo del siglo, nos centraremos en la figura de Electra, y en cómo, acompañada de otras heroínas clásicas, anticipa la representación de la Nueva Mujer de finales de siglo.

Palabras clave: Burlesco, Electra, strong-minded woman, victoriano
\end{abstract}

El teatro burlesco victoriano ha sido objeto de estudio en el ámbito de la filología inglesa desde finales del siglo diecinueve. ${ }^{3}$ Sin embargo, como ya hemos demostrado en otros trabajos, (MonRós-GASPAR, "That's entertainment") la dificultad de clasificación de las obras y de acceso a las fuentes -albergadas en su mayor parte en la colección de manuscritos del Lord Chamberlain en la British Library- han relegado esta parte de la historia del teatro británico al olvido hasta bien entrada la década de 1990.

En este estudio, y a la luz de trabajos previos que demuestran la estrecha conexión entre la historia social de la mujer en el siglo diecinueve y su representación en el teatro popular del momento (Macintosh 2000: 75-99, MonRós-GaSPAR 2009a: 217-307; 2009b: 518-523), analizamos la recreación cómica de la figura de Electra que realiza Francis Talfourd en Electra in a New Electric Light (1859) a partir del estereotipo femenino de la 'New Woman'. Como demostraremos en estas páginas, la influencia de los cánones de

\footnotetext{
Date of reception: 31 May 2010

Date of acceptance: 8 September 2010

2 La investigación realizada para este trabajo se ha financiado con el proyecto I+D financiado por el Ministerio de Ciencia e Innovación con referencia Proyecto FFI2009-12687-CO2-01.

3 Véanse, por ejemplo, FitzGerald (1870) y Adams (1891).
}

Odisea, $n^{\circ} 11$, ISSN 1578-3820, 2010, 223-236 
conducta femenina en la configuración cómica del mito en la Inglaterra decimonónica no es arbitraria. Bien al contrario, responde a un profundo sincretismo estético que se produce en el teatro popular del momento y que transforma la escena victoriana en testimonio fidedigno de la historia social del Imperio Británico. Rodeada de espectacularidad y entretenimiento ligero, la Electra de Talfourd consigue combinar rasgos de configuración del mito en la tragedia griega con imágenes estereotipadas de la mujer victoriana. Con ello, al igual que sucede con recreaciones coetáneas de Medea, Casandra o Antígona realizadas por Robert Brough, Robert Reece o Edward L. Blanchard respectivamente, ${ }^{4}$ Electra se transforma en epítome de la Nueva Mujer que se prefigura desde mediados de siglo.

\section{HEROÍNAS TRÁGICAS Y LA NUEVA MUJER}

La presencia de los clásicos en la educación femenina de la Inglaterra victoriana es tema fundamental en el análisis de los estudios de recepción y de género acerca del rol social de la mujer en el siglo diecinueve. ${ }^{5}$ En una era en la que las fronteras sociales se encuentran marcadas por una preparación intelectual fundamentada en el estudio del latín y del griego (STRAY 1998: 7-30), rescatar de la memoria "the feminine of Homer", en palabras de Isobel Hurst (HuRst 2006), supone incluir en el horizonte de grandes intelectuales del momento el perfil de nombres femeninos desdibujado en muchas ocasiones por la historia y la crítica literaria. Igualmente, y más importante para este trabajo, el estudio de la creación de estereotipos sociales en pluma femenina a partir de figuras míticas de la tradición clásica grecolatina pone de manifiesto no sólo la intelectualidad de estas voces silenciadas sino también usos y costumbres sociales relevantes para un análisis historiográfico de la época.

En 1991, Dolores Mitchell define a la Nueva Mujer de finales de siglo a partir de la figura titánica de Prometeo. Tomando como ejemplo el pincel de Janet Atché (1890-1910), Louise Lavrut (1874-?) y Frances Benjamin Johnston (1864-1952), Mitchell aplica el epíteto a las artistas que representan en sus cuadros a la mujer finisecular en actitudes eminentemente masculinas y que "by appropriating aspects of iconography that had empowered men, they metaphorically stole fire from their society's patriarcal 'gods" (Mitchell 1991: 3). Por su parte, Yopie Prins, en su estudio de 1999 nos habla de la transmisión del verso de Safo en la literatura victoriana, así como de mujeres cuya experiencia vital y artística reproduce a lo largo del siglo el patrón estilístico de la poetisa de Lesbos (Prins 1999: 209-245). Sin embargo, la apropiación de la antigüedad clásica para referir actitudes y comportamientos sociales no es en absoluto algo privativo de la crítica y las artes en general de los siglos XX y XXI sino que ha copado la crítica, el arte y la literatura desde sus comienzos. En lo que respecta a la Inglaterra del siglo XIX, un reciente y revelador estudio de Isobel Hurst, por ejemplo, detalla cómo el magazine misceláneo Woman's World-desde que Oscar Wilde tomara sus riendas como editor en 1887- se convertía en referente de la prensa periódica para la difusión del mundo clásico entre el público femenino a partir de su visión de la actualidad

\footnotetext{
4 Antigone Travestie de Edward L. Blanchard, fue representada por primera vez en 1845 en el New Strand Theatre de Londres; Medea; or the Best of Mothers, with a Brute of a Husband. A Burlesque, in One Act de Robert Brough Robert se estrenó en el Olympic de Londres en 1856; Agamemnon and Cassandra; or, the Prophet and Loss of Troy de Robert Reece, se estrenó en el Prince of Wales de Liverpool en 1868.

5 Véase por ejemplo Jones (1944), Hurst (2006), Monrós-Gaspar (2009), Fiske (2009).
}

Odisea, $n^{\circ} 11$, ISSN 1578-3820, 2010, 223-236 
victoriana desde modelos greco-latinos. Según Hurst, "representations of a wide variety of historical and fictional women operating in both public and private contexts allowed the magazine to appeal to conservative readers as well as feminists" (HuRst 2009: 43). En lo que respecta a estas últimas, Hurst continua sus argumentos exponiendo cómo "Like the dramatic monologue [...], the periodical article could be used to construct an image of an individual female character in the ancient world that implicitly questioned the social norms of Victorian women's lives" (HuRsT 2009: 44). La ambivalencia que expone Hurst -también manifiesta en el teatro burlesco victoriano- es reflejo de la crisis social que provoca la inclusión de la mujer en nuevos espacios públicos, como la educación superior, vetados hasta bien entrado el siglo. ${ }^{6}$ La recepción de la mitología clásica en la Inglaterra victoriana se hace eco de este fenómeno de tal forma que figuras femeninas de la tradición greco-latina aparecen configuradas en puntos opuestos de las bases que establecen el comportamiento femenino del momento. ${ }^{7}$ De este modo, por ejemplo, Casandra toma cuerpo en el ensayo homónimo de 1852 de Florence Nightingale en calidad de la voz de mujer silenciada que debe erigirse como salvadora de la esclavitud femenina, mientras Mary Russel Mitford, en su poema "Joanna's Prophecy" (1811) perpetúa la tradicional imagen victoriana de Casandra como profetisa y carácter marginal de la sociedad decimonónica. ${ }^{8}$

Otro ejemplo llamativo que ilustra claramente esta dicotomía son las dos tendencias antagónicas de la recreación de la figura de Antígona que copan el siglo desde la representación de la Antígona de Mendelssohn en el Covent Garden de Londres en 1845 (HALL \& Macintosh 2005: 316ss). Desde Thomas De Quincey hasta John Gibson y George Eliot, muchos fueron los autores cuya producción literaria se vio inspirada por algunos de los temas esenciales expuestos en esta representación de 1845. El poema de Margaret Sandbach "Antigone" (1850), por ejemplo, ejemplifica la construcción de la honestidad de la heroína que también es evocada por De Quincey (DE QuINCEY 1863: 204-5): ${ }^{9}$

Defiest the mandate. Vainly dost thou prove

The affection, fortitude, and faith divine,

That live in woman's heart, how rich in thine!(SANDBACH 1850: 13)

Algunas recreaciones norteamericanas del mito coincidieron con Sandbach y De Quincey al dar prominencia al sacrificio de Antígona como perpetuador de la figura de "dutiful sister who defied the state to attend to her family and religious conscience" (WINTERER 2001: 70). La prevalencia de la virtuosa Antígona, sin embargo, no consiguió eclipsar por completo el potencial político del mito, y así, en su ensayo "The Antigone and its Moral" publicado en The Leader en 1856, George Eliot relaciona a la hija de Creonte con su gesta

\footnotetext{
6 Para un análisis del fenómeno a partir de los modelos de "revolting daughters" "Girton Girls" y "Advanced Women", recurrentes en la prensa del momento, véase BеЕтнам 1996: 131-141.

Véanse FisKe 2009: 26 por ejemplo, para un análisis al respecto de la figura de Medea y las recreaciones de "Medea in Athens" de Augusta Webster y Medea: A Play in Fragments (1884).

8 Otro claro ejemplo de la tradicional refiguración victoriana de Casandra como profetisa rechazada por la sociedad es el ensayo de William Rathbone Greg Rocks Ahead; or the Warnings of Cassandra (1874).

9 El poema de Sandbach está basado en Antigone discovered over the dead body of her brother (1866), de John Gibson, que continua con la tradición de imágenes de heroínas melodramáticas del siglo dieciocho.
} 
particular contra instituciones anquilosadas de su tiempo al hacer hincapié en la lucha entre el individuo y la sociedad en la que está atrapada Antígona. ${ }^{10}$

En la misma línea, a finales de siglo, cuando el estereotipo de la Nueva Mujer se encuentra ya clara y plenamente definido, el relato corto de Elizabeth Stuart Phelps "Antigone" (1891) denuncia la influencia negativa que ejercen una educación inadecuada y las obligaciones familiares impuestas en el momento en el desarrollo completo de la mujer (WINTERER 2001: 81-4). La relación entre los debates de emancipación femenina y la coexistencia de la Antígona rebelde junto a la Antígona consciente de sus deberes morales es igualmente denunciada por Julia Ward Howe en The Congress of Women de Chicago en 1893:

In some of the comedies of Aristophanes the women's cause is presented in a light intended to provoke ridicule ... For ideal types we must go to those dramatists who deal with the historic and mythic traditions of the past ... In the female characters put upon the stage by Sophocles we can trace within the influence of his friend Socrates, or the sympathy of view which may have formed the bond between them. My present limits will only allow me to speak of two of these characters, Electra and Antigone. Both of these women are rebels against authority. In both of them high courage is combined with womanly sweetness and purity...And in these gracious and more purely feminine types presented by Sophocles, we admire the union of a womanly tenderness with womanly courage (EAGLE 1895: 102-3).

En estas líneas, Howe equipara la fortaleza de Antígona a la de Electra destacando los dos extremos que dan color a la configuración de ambas heroínas a lo largo del siglo diecinueve. La relación entre Electra y el movimiento de emancipación femenina, ampliamente estudiado por Edith Hall, ofrece un gran abanico de reescrituras que, desde la traducción de Charlotte Lennox en 1759 de Le Théâtre des Grecs de Pierre Brumoy (1730) hasta la producción femenina de 1883 de la Electra de Sófocles en Girton Collage (Cambridge), por ejemplo, muestra cómo la hija de Agamenón, de la heroína melodramática incapaz de dar muerte a su madre de mediados del dieciocho se transforma en epítome de la Nueva Mujer de finales de siglo (HaLl 1999: 288-294). ${ }^{11}$

En un periodo en el que el helenismo es fundamento esencial para la creación del gusto estético y de usos y costumbres sociales, no sorprende que las grandes heroínas de la tragedia griega presten su voz a los nuevos ropajes que comienzan a vestir a la mujer desde la década de los cincuenta. En lo que respecta a la Electra de Talfourd, un ingrediente adicional se añade a su sustrato semiótico: la conciencia popular. La esencialidad del teatro burlesco de tema clásico victoriano es su amplio alcance social, que pone de manifiesto el caldo de cultivo que recibe, simultáneamente, las traslaciones de las antiguas tragedias a los grandes géneros dramáticos. En este contexto, a partir del tipo social de la strong-minded woman analizamos a continuación Electra in a New Electric Light (1859), de Francis Talfourd.

\footnotetext{
10 Algunos sectores de la crítica ven paralelismos entre Maggie, de The Mill on the Floss y Antígona. Véase FiSKE 2009: 220 para una bibliografía detallada entorno a George Eliot y la figura de Antígona.

11 Véase REID 1993: i. 373.
} 
FRANCIS TALFOURD, STRONG-MINDED WOMEN Y ELECTRA IN A NEW ELECTRIC LIGHT (1859)

La frase argumental que estructura la figura de Electra en la tragedia griega es la venganza de la muerte de su padre, Agamenón, de manos de Clitemnestra, su propia madre, y Egisto, su amante. Tras diez años de contienda en Troya, Agamenón, rey de Argos regresa a su hogar con sus tropas y con Casandra, princesa troyana que toma como botín de guerra. Su regreso pronto se torna en tragedia cuando Clitemnestra y Egisto asesinan al rey y a su nueva concubina. Tras el crimen, Electra espera el regreso de su hermano Orestes y la venganza de las muertes. Variaciones del argumento se encuentran en Coéforas de Esquilo, Electra de Sófocles y la obra homónima de Eurípides. ${ }^{12}$ Sin embargo, ninguno de los conflictos trágicos de estas tres Electras clásicas es adoptado completamente por el personaje de Talfourd, sino que la versión burlesca del mito se fundamenta únicamente en la atrevida voz de Electra frente al poder.

Electra in a New Electric Light se estrenó en el Theatre Royal Haymarket de Londres en 1859 (TALFOURD 1859: 2). El título de la pieza hace alusión al uso de la lámpara de arco de carbón por primera vez en Inglaterra por parte de Paul Taglioni en el nuevo ballet, ${ }^{13}$ The Lost Pleiade, que se estrenó con gran éxito en el Her Majesty's Theatre de Londres en 1849 (Hall \& Macintosh 2005: 360-1). En Talfourd, el papel de Electra fue representado por E. Weekes, según informa la edición de la obra publicada en la Lacy's Acting Plays Collection en 1859 (TALFOURD 1859:3), ${ }^{14}$ conocida actriz del momento que ya había aparecido en obras como The Beaux Stratagem en el Haymarket Theatre en diciembre de $1858 .{ }^{15}$ En el dramatis personae, Electra aparece descrita como "the strong-minded daughter of Agamemnon and Clytemnestra, who looks forward to the removal of her present Sovereigns as the termination of her present sufferings" (TALFOURD 1859: 3). El estereotipo de la strong-minded woman copó las artes, la literatura y la prensa británicas desde mediados del diecinueve como precedente de lo que en las últimas décadas del siglo sería la Nueva Mujer. El término Nueva Mujer fue utilizado por primera vez por Sarah Grand en un ensayo publicado en 1894 en el North American Review haciendo alusión al nuevo resurgir de la mujer en la sociedad del momento. ${ }^{16}$ Tal y como sugiere Nelson:

A stereotyped image of the New Woman quickly took hold on the public imagination. She was educated at Girton College, Cambridge, rode a bicycle, insisted on rational dress, and smoked in public: in short, she rejected the traditional role for women and demanded emancipation... The New Woman, arguing that the separate spheres ideology was a

\footnotetext{
12 Véase BAÑUls, CRESPO y Morenilla (2006) para un estudio pormenorizado de las mismas y su relación con recreaciones hispanas del mito. Véanse Morenilla (2003b) y WheEler (2003) para un estudio de la figura de Electra en la Antigüedad.

13 Véase ILN 14, no.369 (5 May 1849) 293.

14 Además del manuscrito British Library Add. MS 52982, C, albergado en la Lord Chamberlain's Plays Collection, ésta es la única edición que se conserva de la obra desde su representación en 1859.

15 Véase The Players: the Abstract and Brief Chronicles of the Time, 1, no. 17 (21 April 1860), 134.

16 Véase "The New Aspect of the Woman Question," North American Review, 158 (March 1894), 448 como aparece editado en NELSON 2000: 142, para una descripción pormenorizada del término Nueva Mujer.
} 
construct of society and culture rather than biological mandate, demanded that women be given the same opportunities and choices as men (NELSON 2000: ix).

La expresión fue utilizada tanto por defensores como por detractores del modelo y pronto se convirtió en tópico recurrente en los escritos del momento.

Esta Nueva Mujer coincide con las strong-minded women en una gran cantidad de lugares comunes que la transforman en heredera directa del tipo social que representa Electra en Talfourd. Ambos referentes, la Nueva Mujer y la strong-minded woman, eran fácilmente reconocibles para el público del momento ya que, entre otros aspectos, y como defiende Ellen Jordan, se encontraban relacionados con un gran número de profesiones emergentes cada vez más relacionadas con el espacio social ocupado por la mujer (JORDAN 1999:87-144). Una controversia de tanta actualidad como el surgimiento de las strong-minded women no podía escapar a la pluma burlesca y pronto se convirtió en un blanco cómodo para la prensa satírica y el teatro contemporáneos. ${ }^{17}$ La presencia de las strong-minded women en el teatro burlesco de tema clásico del siglo diecinueve es recurrente. Cabe destacar, sin embargo, que las osadías del modelo de mujer determinada que refleja este teatro se encuentran más vinculadas a su aniquilación del rol del ángel del hogar que desafía las convenciones establecidas en manuales de conducta que al activismo por la liberación femenina o al ejercicio de profesiones y actitudes hasta el momento eminentemente masculinas. Ejemplo de ello son la Alcestis del propio Talfourd y la Clitemnestra de Robert Reece que comentamos brevemente a continuación.

En 1850, Francis Talfourd estrena en el Strand Theatre de Londres Alcestis, the Original Strong-Minded Woman con Mrs. L. Murray en el papel principal. Como apuntan Hall y Macintosh, la domesticación burlesca realizada por Talfourd centra la configuración del personaje en problemas contemporáneos tales como la situación de la mujer dentro de la institución del matrimonio cuando la legalización del divorcio todavía se encontraba en un estado embrionario (HALl \& MACINTOSH 2005:433-8). Al contrario que su padre, Francis Talfourd no fue un ferviente activista de los derechos de la mujer. ${ }^{18}$ Sin embargo, el soliloquio clásico de su Alcestis debió sin duda suscitar la empatía del público femenino con la heroína al evocar cuestiones tan candentes como la desigualdad de géneros dentro del matrimonio:

(Alcestis advances with the two children)

Alcestis:

Oh! sun, and moon, and stars! oh, day and night!

Oh every thing above an inch in height!

Oh Day! as black as black of Day and Martin,

\footnotetext{
17 Como ejemplo de la presencia de las strong-minded women en la prensa del momento, véase TAYLOR, W. C., "The Strong-minded Woman" The St. James's Magazine, 15 (1866), 350-364; "The Ladies of the Creation: or How I was Cured of Being a Strong-Minded Woman" Punch 24 (1852) x-xii, xiv, xvi, xviii; "The Strong-minded Women"Fraser's Magazine for Town and Country, 68 (November 1863), 667-678.

18 Sir Thomas Noon Talfourd, padre de Francis Talfourd promovió, entre otras la Infant Custody Act (1839) en el parlamento, que otorgaba la custodia de los hijos con edades inferiores a siete años a la madre en casos de divorcio. Edith Hall, 'Talfourd, Sir Thomas Noon (1795-1854)', Oxford Dictionary of National Biography, Oxford University Press, 2004 [http://www.oxforddnb.com/view/article/26951, accessed 3 May 2010].
} 
To what infernal realms must I be starting!

Oh bed! -beg pardon- nuptial couch, I mean,

'Twere green, though, to regret now Gretna Green.

Else might I ask, were not the question idle,

Why was I ever saddled with this bridal?

Or why -but these, alas, are whys too late.

Did I with such milksop link my fate!

Why at the altar did we join our hands?

Why Hymen e'er unite us in his bands?

Those bands which ne'er have paled thee, heavy waits,

A-merry-key in our united states?

Why was my heart to be with such a spoony un,

A wretched picture of a poor heart union?

For life with him was nothing but a curse,

And though I took him 'for better or for worse.'

The world can't surely wonder I forsook him, for

I found him such a deal worse than I took him for.

Oh, parent hearth! oh, earth, air, fire, and water!

$\mathrm{Oh}$, son in petticoats and unmarried daughter!

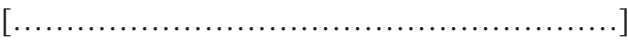

When I am gone? or who supply the stitches

That may be wanting in his infant -trousers?

And when in youth his jacket he outwears,

And sows his wild oats, whe's to sow his tares?

I might, if I had space, expatiate-

Alas! though, I've no room to ruminate,

Still les, as I die early, to di-late!

So I have done -another observation

Would be entire supererogation.

My life, 'tis clear, no words of mine can save.

And I must pass at once from 'gay to grave!'

That bourne from which each traveller born soon learns. (TALFOURD 1859:16-17.)

Siguiendo de nuevo el referente Talfourdiano, Robert Reece estrena en el Teatro Prince of Wales de Liverpool en 1868 Agamemnon and Cassandra; or, the Prophet and Loss of Troy (1868), con Clitemnestra en el papel de:

The original strong-minded woman, with only one weakness, viz. for Aegisthus - the founder of the Queen in 'Hamlet,' 'Lady Macbeth,' Mrs. Caudle, \&c., a woman who sticks at nothing (save her enemy!) and altogether a type not to be followed by British matrons (REECE 1868: 5).

En la obra de Reece, y en marcado contraste con Egisto y Agamenón, Clitemnestra es explícitamente resuelta e inteligente, reflejando la tendencia habitual de refiguración del mito en el siglo diecinueve británico representada, por ejemplo, por William Thackeray o Edward Nolan (MacinTOSH 2005: 139-162). Reece toma como referente la cómica relación 
entre Lady Macbeth y su esposo en la conocida recreación burlesca de la tragedia Shakesperiana del propio Talfourd, Macbeth Travestie, representada en 1847 en la famosa regata de Henley en Oxford (Mackinnon 1910: 21-22). ${ }^{19}$ Paralelismos entre la configuración de los personajes principales femeninos en ambas revelan un modelo de esposa, como la Alcestis de Talfourd, nada apto para los manuales de conducta del momento:

Lady M. Why did your bring those daggers from their [places?

Go, take them back, and smear the sleepers' faces

With blood.

Macb. (doggedly) No: come you know, I've done one

[murder;

That's quite enough, and I sha'nt go no furder.

Lady M. Don't leave the job unfinished, come now, don't;

[Go;

Mab: If I do I'm -, never mind, I wont!

Lady M. Be mine the task, since you the courage lack;

Give me the daggers, I shall soon be back.

[Exit (R) (TALFOURD 1850: 13)

Agam. What do you do here?

Aegis. Do! Why I'm done! I'm boiled.

'Twas she who put me here.

Clyt. (drawing dagger) Nay, if you blab,

'Tis Clytemnestra's hand must give the stab.

(rushes at AGAMEMNON, is seized by PHYLAX,

CHORAGUS and TALTHYBIUS-AEGISTHUS is held by

the FURIES -TROPHE tips over into the bath and is

brought out by EROS -CASSANDRA delighted.) (REECE 1868: 35)

Como sucede en la configuración de estas figuras míticas, ${ }^{20}$ los estereotipos empleados y desarrollados por Talfourd en Electra se fundamentan en concepciones establecidas en torno a estos modelos de mujer poco ortodoxos a partir de las strong-minded women. Así, en Electra la hija de Agamenón interpreta el rol de joven leguaraz poco interesada en afeites personales y aventuras amorosas hasta que se cumple su único y primordial objetivo,

\footnotetext{
19 Durante sus años en Oxford, entre 1857 y 1864 Robert Reece también perteneció a la compañía de Oxford Amateurs (MACKINNON 1910: 29). A pesar de no coincidir cronológicamente con la representación de la versión de Macbeth de Talfourd, probablemente ésta fuese ampliamente comentada dentro del grupo dado el éxito de la misma. La relación entre el mito de Clitemnestra y Lady Macbeth ha sido ampliamente estudiada. Véanse, por ejemplo, JAMESON 1832: ii. 304-245 y MuiR [1977] 2005. Véase Poole 2004: 98-102 para la relación entre las dos figuras en la Inglaterra Victoriana. Véase Morenilla (2003a) para un estudio de la determinación de Clitemnestra en la antigüedad.

20 Una tercera strong-minded woman, aunque con menos relevancia, que encontramos en el teatro burlesco victoriano de tema clásico es Kautatarta, descrita en la lista de personajes de Perseus and Andromeda de William Brough como "a strong-minded female citizen of Seriphos, by far the better half of Dullas" e interpretada por Nelly Moore. Perseus and Andromeda; or, the Maid and the Monster fue representada por primera vez en el Royal St. James' Theatre de Londres el 26 de diciembre de 1861.
} 
la venganza política de la muerte de su padre y el derrocamiento de los dos culpables que ostentan el poder. Como veremos a continuación, Talfourd configura de forma satírica el personaje como estereotipo de strong-minded woman, lo que podría ser un claro indicativo de su alineamiento con los convencionalismos del momento. No obstante, otros aspectos de la pieza inclinan la balanza hacia el desafío de los mismos, lo que pone una vez más de relieve la importancia del teatro burlesco victoriano como reflejo artístico de las ambivalencias sociales de la Inglaterra decimonónica.

La representación de la cabellera femenina, tan estudiada y tan atractiva al gusto victoriano (GitTer 1984; Bornay 1994; OfeK 2009), es el punto de partida desde donde analizamos esta configuración burlesca del personaje de Electra. Como hemos estudiado en otros trabajos a propósito de la representación de la figura de Casandra (MonRós-GASPAR 2006), la estrecha relación entre literatura y pintura en la representación femenina a lo largo del siglo diecinueve es latente en el teatro popular del momento. De este modo, femme fatales, fallen women y strong-minded women aparecen con cabellos oscuros y despeinados mostrando su peligro y displicencia hacia los cánones establecidos (OFEK 2009: 65, 145, 150-1). En Talfourd, el tópico se repite y se completa, como también es habitual, con unos ropajes desaliñados. De este modo, el autor consigue trasladar la oposición sofoclea entre Crisótemis y Electra a debates contemporáneos, y lo que en la tragedia griega revela una diferencia de trato entre las dos hermanas por parte del poder imperante, en la recreación burlesca se transforma en una pugna entre los convencionalismos del 'ángel del hogar' y la heterodoxia. De este modo, el interés político de Electra se manifiesta en una apariencia 'poco femenina' relacionada con la caracterización de mujeres victorianas intelectuales cuyo acceso al conocimiento se traduce en locura e incluso histeria:

Aegist.

She may be broken but will never mend herself

Besides her dress is -where got I can't tell,

Apparel quite without a parallel:

Wherein she wanders forth at early morn,

Unkempt, uncinctured, with her stockings torn,

In her hand offerings, in her eye a fear

To imbrue her fathers lamen-table beer.

Chrysothemis is of another sort,

On politics she never wastes a thought.

Clytem.

I doubt if she's a thought to waste, unless

It be upon the fashion of her dress

Thus different ways, each in her duty flags

One's over dressed, the other done to rags.(TALFOURD 1859: 8)

[.......]

Enter ELECTRA, L., her hair dishevelled, her dressed torn

and disarranged, shoes unsandaled and down at heel.

Electra. Another day has passed, and yet another

Brings with its light no tidings of my brother.

While poor Electra, wearied of expecting 
By all neglected, and herself neglecting,

Resembles much as classic heroine can

The well know slip shod, Good-for-nothing Nan

These locks of gold when servants on me waited

Used to be carefully electra-plaited.

Now all dis- Sheffield down my shoulders flow-

No friendly comb'll make them comb il faut;

Where a 'deserted auburn' they remain,

I fear not 'loveliest even of the plain. (TALFOURD 1859:12)

Si bien esta configuración del personaje es análoga a la satirización grotesca de modelos femeninos poco ortodoxos, es necesario puntualizar que únicamente cuando el regreso de Orestes es efectivo y la venganza de los dos hermanos inminente, vuelve Talfourd a caracterizar a la heroína según los cánones de belleza establecidos. Por añadidura, también cabe señalar cómo, a pesar de ser Orestes - siguiendo el modelo sofocleo y también los cánones sociales del momento- quien lleva a cabo de forma activa la venganza de la muerte de Agamenón, Electra es el cerebro ardid de la misma. La fortaleza de su carácter, en manifiesta oposición al canon representado por Chrysothemis, se hace patente en el diálogo que establece con Clytemnestra en I.ii:

\section{Electra:}

It was not I-so that reproach may spare itWho was the first to tap my father's claret.

Have you no tributes to bestow? I pity you.

Clytem:

I wish you'd try boots, for your shoes don't fit you.

Electra:

E'en in my shoes my sympathy's revealed

With him whose soul you sent down unan-healed.

Clytem:

Still the old tune and words! (TALFOURD 1859:13).

También en los consejos a su prima en esta obra, a quien insta a defender su causa con un discurso bélico poco apropiado para una mujer de su alcurnia: "If then you don't her indignation fear,/ Enlist in my cause as a Volunteer." (TALFOURD 1859: 12) 21 $^{2}$

El personaje de Electra de Talfourd, como strong-minded woman, representa en definitiva a una joven de carácter, interesada en la política en la medida en la que ésta contribuye a su lucha contra un poder establecido corrupto, con un discurso en ocasiones bañado de referencias culturales a espacios masculinos, y una apariencia física análoga a los cuerpos femeninos victorianos relegados al margen por su amenaza al canon imperante. Al contrario que la Medea de Brough, sin embargo, el texto de Talfourd no exhibe una clara intención feminista. Por este motivo, la configuración del personaje de Electra desde el humor como preludio a la Nueva Mujer de finales de siglo es si cabe más relevante. En primer lugar, en

21 Nótese la variación con respecto a las fuentes clásicas. En Talfourd, Chrysothemis es prima y no hermana de Electra para forzar su unión con Orestes al final de la pieza. 
calidad de manifestación artística popular, demuestra cómo el debate acerca de la cuestión femenina se extiende, desde mediados de siglo, más allá de las fronteras de los círculos políticos e intelectuales. En segundo lugar, estudiada en el contexto de recepción de otras figuras míticas femeninas bajo el mismo modelo, pone de manifiesto la actualidad y la importancia del mundo clásico en un siglo de la historia británica que forja gran parte de los fundamentos de la cultura contemporánea.

A propósito de estos dos aspectos, es necesario hacer alusión a otras dos recreaciones cómicas del mito de la tradición española que coinciden en parámetros similares: Electroterapia (1901) de Gabriel Merino y Pichilo y Alerta! (1901) de Federico Rodríguez Escacena y Rafael Muñoz Esteban. Las dos piezas son versiones de la afamada tragedia de Benito Pérez Galdós, Electra, que fue estrenada con gran éxito el mismo año en el teatro Apolo de Madrid. La deuda galdosiana a Sófocles, Eurípides o Esquilo ha sido objeto de controversia entre hispanistas, la gran mayoría de los cuales considera la obra una tragedia nacional con únicamente tenues guiños hacia sus precedentes clásicos. Los vínculos con las fuentes griegas son tan imperceptibles en la tragedia galdosiana (excepto en el título y algunas alusiones y rasgos menores) que desaparecen por completo en las dos recreaciones de Merino y Rodríguez Escacena. Nos referimos sin embargo a ambas en este trabajo puesto que son las dos únicas obras que hemos encontrado hasta ahora en nuestra investigación, derivadas de un texto clásico acerca de la figura de Electra, y fechadas en las décadas clave del movimiento feminista en Europa, en las que la configuración del personaje femenino principal ataca con acciones y discurso el canon de comportamiento femenino establecido. La aportación de estas obras a nuestro estudio supone la contextualización de la Electra de Talfourd en una estética más amplia que la especificidad del momento de representación, y que nos devuelve a las líneas con las que comenzamos este estudio. Tras desvestir a la Electra griega del conflicto trágico, transformar sus rasgos de parentesco y situarla en un espacio y lugar de acción totalmente ajenos a Esquilo, Eurípides o Sófocles, el único rastro que queda del personaje clásico, como en la Electra de Talfourd, es su configuración a partir de la oposición a cánones femeninos preestablecidos. Así pues, el análisis de Electra in a New Electric Light desde el prisma de las strong-minded women no sólo arroja luz sobre esta pieza en calidad de ejemplo paradigmático de un fenómeno generalizado en el teatro burlesco victoriano, sino que también permite cerrar estas páginas con un debate abierto para futuros trabajos: la función social del humor en la construcción de modelos sociales y la transmisión y la recepción de la antigüedad clásica en el contexto de la cultura de masas.

\section{REFERENCIAS BIBLIOGRÁFICAS}

Adams, W.D. 1891. A Book of Burlesque. Sketches of English Stage Travestie and Parody. London: The whitfriars library of wit and humour.

Bañuls, J.V., P. Crespo y C. Morenilla. 2006. Electra de Sófocles y las primeras recreaciones hispanas. Bari: Levante Editori.

Beetham, M. 1996. A Magazine of her own?: Domesticity and Desire in the Woman's Magazine, 1800-1914. New York: Routledge.

Odisea, ${ }^{\circ} 11$, ISSN 1578-3820, 2010, 223-236 
Blanchard, E. L. 1845. Antigone Travesty (British Library Add. MS 42982, F 16673).

Bornay, E. 1994. La cabellera femenina. Un diálogo entre poesía y pintura. Madrid: Editorial Cátedra.

Brough, R. B. 1856. Medea; or the Best of Mothers, with a Brute of a Husband. A Burlesque, in One Act. London: T. H. Lacy 1861. Perseus and Andromeda. London: T.H. Lacy.

DeQuincey, T. 1863. The Art of Conversation and Other Papers. Edinburgh: A and C Black.

Eagle, M. K. O. 1895. The Congress of Women Held in the Woman's Building, World's Columbian Exposition (Chicago). The Gerritsen Collection of Aletta H. Jacobs. College University Library. 15 Oct. 2007. <http://gerritsen.chadwyck.com>

Ецiot, G. 1856. “The Antigone and Its Moral”. The Leader, (29 March), 306.

FISKE, S. 2009. Heretical Hellenism: Women Writers, Ancient Greece, and the Victorian Popular Imagination. Athens, Ohio: Ohio University Press.

Fitzgerald, P. 1870. Principles of Comedy and Dramatic Effect. London: Tinsley.

Frasers Magazine for Town and Country

GitTer, E. 1984. “The Power of Women's Hair in the Victorian Imagination”. PMLA 99, V: 936-954.

Greg, W. R. 1874. Rocks Ahead; or, the Warnings of Cassandra. London: Trübner \& Co.

Hall, E.1999. "Sophocles' Electra in Britain." Sophocles Revisited: Essays Presented to Sir Hugh Lloyd-Jones. Ed. J. Griffin. Oxford: Oxford University Press. 261-306.

y F. Macintosh. 2005. Greek Tragedy and the British Theatre 1660-1914. Oxford: Oxford University Press.

Hurst, I. 2006. Victorian Women Writers and the Classics. The Feminine of Homer. Oxford: Oxford University Press. Ilustrated London News.

Jameson, A. 1832. Characteristics of Women, Moral, Poetical, and Historical, 2 vols. London: Saunders and Otley.

Jones, F. P. "The Role of the Clasics in the Emancipation of Women" The Classical Journal. 39, VI: 326-342.

Jordan, E. 1999. The Women's Movement and Women's Employment in Nineteenth Century Britain. London: Routledge.

Macintosh, F. 2000. "Medea Transposed: Burlesque and Gender on the Mid-Victorian Stage." Medea in Performance 1500-2000. Eds. E. Hall, F. Macintosh y O. Taplin Oxford: Legenda. 74-99.

Mackinnon, A. 1910. The Oxford Amateurs. London:Chapman \& Hall, Ltd. 
Merino Pichilo, G. (1901) 2003. "Electroterapia: humorada en un acto y tres cuadros original y en verso, parodia del drama en cinco actos 'Electra"'. Biblioteca Virtual Miguel de Cervantes. Alicante. 30 Abr. 2010. <http://www.cervantesvirtual.com/ servlet/SirveObras/paro/01372786433571623089024/index.htm>

Mitchell, D. 1991. "The New Woman as Prometheus: Women Artist Depict Women Smoking”. Woman's Art Journal. 12, 1: 3-9.

Monrós-Gaspar, L. 2006. "Rossetti configurando una Cassandra prerrafaelita." El teatro greco-latino y su recepción en la tradición occidental. Ed. Fr. De Martino y C. Morenilla. Bari: Levante Editori. 405-29.

2009a. Classical Myths on the Victorian Popular Stage: the Figure of Cassandra. Tesis Doctoral. Valencia: Universitat de València.

2009b. "Diosas cómicas y libertadoras: modelos de mujer en el teatro victoriano" Identidades femeninas en un mundo plural. Ed. Elena Jaime de Pablos. Sevilla: Arcibel Editores. 517-524. prensa).

. "That's entertainment". Quaderns de Filologia. Universitat de València. (en

Morenilla, C. 2003a. "La aristeia de una mujer: Clitemnestra domina la escena." Mitos femeninos de la cultura clásica: creaciones y recreaciones en la historia y la literatura. Eds. R.M. Cid López y M.González González. Oviedo: KRK ediciones. 123-149. 19-36.

2003b. "La irascible Electra." Revista cuatrimestral de humanidades 39:

Muir, K. (1977) 2005. The Sources of Shakespeare's Plays. London: Methuen.

Nelson, C. C. 2000. A New Woman Reader: Fiction, Articles, and Drama of the 1890s. Peterborough, ON: Broadview Press.

Oxford Dictionary of National Biography. Online Edition.

OFeK, G. 2009. Representations of Hair in Victorian Literature and Culture. Burlington: Ashgate Publishing.

Poole, A. 2004. Shakespeare and the Victorians. London: Arden Shakespeare.

PrINS, Y. 1999. Victorian Sappho. Princeton: Princeton University Press.

Reece, R. 1868. Agamemnon and Cassandra; or, the Prophet and Loss of Troy, Liverpool.

ReID, J.D. 1993. The Oxford Guide to Classical Mythology in the Arts (1300-1990s). Oxford: Oxford University Press. 2 vols.

Rodríguez Escacena, F. y R. Muñoz Esteban (1901) 2003. “¡Alerta!: pseudos-parodia político musical del famoso drama de D. Benito Pérez Galdós "Electra” en un acto dividido en cuadros, en verso."Biblioteca Virtual Miguel de Cervantes. Alicante. Biblioteca Nacional. Madrid. 30 Abr. 2010. 
$<$ http://www.cervantesvirtual.com/servlet/SirveObras/paro/35738307878251619976613/ index.htm>

St. James' Magazine.

Stray, C. 1988. Classics Transformed. Schools, Universities, and Society in England, 1830-1960. Oxford: Oxford University Press.

TAlfourd, F. 1850. Macbeth Travestie. Oxford: E.T. Spiers. . 1859. Electra, in a New Electric Light. London: Lacy's Acting Edition.

TAYlor, W. C., 1866. “The Strong-minded Woman” The St. James's Magazine, 15, 350-364.

Wheeler, G. 2003. "Gender and Transgression in Sophocles' Electra" The Classical Quaterly, New Series 53, 2: 377-388.

Winterer, C. 2001. "Victorian Antigone: Classicism and Women's Education in America, 1840-1900” American Quarterly 53, 1: 70-93. 